\title{
On IGP Link Weight Optimization for joint Energy Efficiency and Load Balancing Improvement
}

\author{
Frederic Francois $^{\mathrm{a} \dagger}$, Ning Wang ${ }^{\mathrm{a}}$, Klaus Moessner ${ }^{\mathrm{a}}$, Stylianos Georgoulas $^{\mathrm{a}}, \mathrm{Ke}_{\mathrm{Xu}}^{\mathrm{b}}$ \\ ${ }^{a}$ Centre for Communication Systems Research, University of Surrey, Guildford GU2 7XH, UK \\ ${ }^{b}$ Department of Computer Science, Tsinghua University, Beijing, 100084, China \\ †Corresponding author. Email: f.francois@surrey.ac.uk. Mobile: +447733156024
}

\begin{abstract}
The energy consumption of backbone networks has risen exponentially during the past decade with the advent of various bandwidthhungry applications. To address this serious issue, network operators are keen to identify new energy-saving techniques to green their networks. Up to this point, the optimization of IGP link weights has only been used for load-balancing operations in IP-based networks. In this paper, we introduce a novel link weight setting algorithm, the Green Load-balancing Algorithm (GLA), which is able to jointly optimize both energy efficiency and load-balancing in backbone networks without any modification to the underlying network protocols. The distinct advantage of GLA is that it can be directly applied on top of existing link-sleeping based Energy-aware Traffic Engineering (ETE) schemes in order to achieve substantially improved energy saving gains, while at the same time maintain traditional traffic engineering objectives. In order to evaluate the performance of GLA without losing generality, we applied the scheme to a number of recently proposed but diverse ETE schemes based on link sleeping operations. Evaluation results based on the European academic network topology GÉANT and its real traffic matrices show that GLA is able to achieve significantly improved energy efficiency compared to the original standalone algorithms, while also achieving near-optimal load-balancing performance. In addition, we further consider end-to-end traffic delay requirements since the optimization of link weights for load-balancing and energy savings may introduce substantially increased traffic delay after link sleeping. In order to solve this issue, we modified the existing ETE schemes to improve their end-to-end traffic delay performance. The evaluation of the modified ETE schemes together with GLA shows that it is still possible to save a significant amount of energy while achieving substantial load-balancing within a given traffic delay constraint.
\end{abstract}

Keywords: Green networks; link-weight setting; energy-efficiency; load-balancing; packet delay

\section{Introduction}

Computer networks have been consuming an increasing amount of energy because of their greater pervasiveness due to the need to support new network applications such as video based services and cloud computing. Nowadays, network operators are keen to identify new efficient ways of greening their networks so as to reduce both their operational costs and environmental impact. By 2020, European Telecoms are expected to consume around 35.8TWh, a rapid rise from the current 21.4TWh consumed per year, if greener networks are not deployed [1]. More specifically, the share of backbone networks in the total network power consumption will increase from $10 \%$ to $40 \%$ by 2020 if no green actions are taken. This rapid change is mainly driven by the ever increasing popularity of bandwidth-hungry applications over the Internet. In order to tackle the rapid increase in the energy consumption of backbone networks, different Energy-aware Traffic Engineering (ETE) schemes have been proposed in the last few years [2][3][8][9][11][12]. These schemes apply different sleep reconfiguration and transmission rate adaptation techniques at network devices to achieve enhanced energy efficiency. One typical strategy is to use only a subset of network devices for carrying customer traffic when the traffic volume is low during off-peak time. This allows other network devices to have the opportunity to save energy, e.g. through sleeping. As long as the reduced network capacity is sufficient for handling the traffic demand, energy can be saved without causing service deterioration to end users.

On the other hand, network operators traditionally employ traffic engineering (TE) schemes [4] for the sole purpose of load-balancing because this was their main concern in the past before the energy efficiency issue also became a concern. Traditionally, the ultimate objective of load-balancing is to reduce the Maximum Link Utilization (MLU) in the network through optimized distribution of traffic. This reduction in MLU allows the networks to offer better Quality-of-Service (QoS) assurance and also to efficiently handle unexpected traffic surges. However, conventional load-balancing and ETE are intuitively conflicting with each other in network configurations since load-balancing attempts to distribute the traffic as

\footnotetext{
${ }^{*}$ A preliminary version of this paper has been accepted for publication at the IEEE/IFIP Networking 2013 Conference. The conference paper version is provided as supporting material for this submission.
} 
evenly as possible while ETE algorithms attempt to concentrate traffic on the smallest feasible subset of active devices (e.g. network links) in order to allow other elements to sleep. To intelligently resolve such a tussle, a new ETE scheme called Green Load-balancing Algorithm (GLA) is proposed in this paper. The novelty of such a scheme is that it jointly optimizes the load-balancing and energy efficiency in a holistic manner. GLA achieves such objectives by optimizing the Interior Gateway Protocol (IGP) link weights in order to maximize energy saving gains through link sleeping, while maintaining, or even further improving the load-balancing performance of the residual working topology. This is in salient contrast to the conventional single-objective IGP link weight setting schemes that optimize for load-balancing only and therefore do not efficiently provide opportunities for link sleeping operations. In summary, our contribution is a practical solution that opens a new dimension of energy efficiency optimization, but without sacrificing traditional traffic engineering performance in plain IP routing environments.

The rest of this paper is organized as follows: the related work is described in detail in Section 2 and an illustrative example to highlight the concepts and reasoning of GLA is given in Section 3. In Section 4, the problem formulation of optimizing link weights for both load-balancing and energy efficiency is presented. A short description of the three ETE schemes that are used in this paper to evaluate the performance of GLA is also provided in Section 4. An in-depth description of the GLA mechanism is given in Section 5 and GLA is evaluated with the help of the Point-of-Presence representation of the European academic network GÉANT and its real traffic matrices in Section 6. GLA is shown to substantially improve both the load-balancing and energy efficiency of the three chosen existing ETE schemes. In Section 7, GLA is further customized for a specific ETE scheme and is shown to improve even more the performance of this ETE scheme. This shows that GLA can be regarded as a generic enough technique that can be further customized based on the specifics and particularities of the ETE schemes it is applied on. In Section 8, the maximum propagation delay of the different ETE schemes is evaluated. Since, as will be shown, this delay can be excessive as a result of their operations, the three existing ETE schemes are modified in Section 9 so that they can respect a constraint on the maximum propagation delay. The new delay-aware ETE schemes are evaluated against their original counterparts in Section 10. Finally, the paper is summarized and some directions for future work are provided in Section 11.

\section{Related Work}

A number of strategies are currently being followed to reduce the energy consumption of backbone networks. The first strategy is the use of more energy-efficient hardware in network equipment. Energy efficiency of the hardware can be improved by employing lower power circuit design at the physical level ([9][10][11]) and using dynamic frequency and voltage scaling at the functional level ([12][13][14]). Secondly, it is also possible to make use of greener networking protocols. For example, green protocols can enable a proxy to take over the functionality of a number of network devices which are then put into sleep mode ([15][16][17]). Such an approach has been illustrated in [17] where a router can transparently aggregate the virtual links on several line cards onto a smaller number of line cards so as to allow the remaining line cards to go to sleep. Another example is to modify the OSPF protocol by making a subset of routers use the routing table of other routers, rather than calculating their own, so that fewer active links are needed [15]. The aforementioned two strategies can be considered as disruptive because they require either a replacement of existing network equipment or manufacturers releasing new firmware. As such, these strategies can be viewed as long-term solutions to green the network because it will take a long time for wide deployment. In the meantime, there are already-available evolutionary strategies ([18][19][7]) which can be directly implemented in legacy network systems. One such strategy is the collection of Energyaware Traffic Engineering (ETE) schemes.

The field of ETE started with the seminal work in [20] where the authors explore how traffic can be re-routed so as to promote energy efficiency in network devices. Since then, numerous ETE schemes have been developed and summarized in the two recent surveys of [2] and [3]. The different ETE schemes can be classified as either offline or online. Offline ETE schemes ([6][7][8][21][22][23][24]) usually compute static network configurations based on forecasted traffic matrices. These schemes offer the advantage of practical deployment based on existing network devices and protocols. Another advantage of offline schemes is that they are able to achieve higher energy efficiency and traffic performance due to the use of global optimization techniques thanks to the availability of a complete view on the network state. Offline ETE schemes are usually easy to deploy on top of already standardised protocols, and therefore, they are more likely to be adopted by network operators.

In contrast to offline ETE paradigms that are relatively vulnerable to unpredictable traffic dynamics, online ETE schemes $([20][25][26][27][28][29][30])$ continuously monitor the state of the network at a shorter timescale which allows them to perform on-the-fly re-configurations of the network. They are more agile to unexpected traffic dynamics, but they often suffer from limited knowledge of the network state when computing a new configuration locally. Similar to plain online traffic engineering (TE) techniques, online ETE mechanisms rely on accurate network monitoring and face additional challenges such as network stability and necessary requirements on protocol extensions. This makes the online ETE solutions more difficult to be deployed in operational networks compared to their offline counterparts.

It is worth mentioning that most existing offline ETE schemes ([7][8][21][22][23][24]) are based on IGP routing. When these offline ETE schemes perform link sleeping in order to reduce the energy consumption of backbone networks, they do 
not consider the scenario of manipulating the IGP link weights in order to optimize their performance. In contrast, our proposed GLA scheme aims at the holistic improvement of both energy efficiency and load-balancing by setting IGP link weights such that this yields substantially improved network performances compared to the plain ETE schemes.

\section{An Illustrative Example of GLA}

In order to illustrate the basic concept of GLA-based link weight optimization for both load-balancing and energy efficiency, we use the small example network topology in Fig. 1 with the indicated link capacities and IGP weight settings. The aim of such an example is to illustrate how IGP link weights can be manipulated in order to create opportunities for more links to sleep, but without affecting the load-balancing requirements. For simplicity and clarity, we use an "incomplete" uni-directional graph, but certainly such an idea is also applicable to real network topologies with full bidirectional connectivity. First of all, it can be observed that there are only two links which can be put to sleep without causing the network topology to lose full connectivity: namely links $C \rightarrow D$ and $A \rightarrow D$. We first consider the case where the set of link weights is non-optimized as shown on the left, and then the case where the link weights are optimized on the right side of the figure.

Given a simple illustrative traffic matrix composed of only traffic demands between two Source-Destination pairs: $C-D$ and $A-D$ of 30 and 75 units respectively, the traffic demand $C-D$ goes through path $C \rightarrow D$ with a link utilization of $60 \%$. For the traffic demand $A-D$, path $A \rightarrow D$ is used with a link utilization of $75 \%$. The original MLU in this scenario is therefore $75 \%$. Based on the given traffic demands, we can follow conventional techniques such as [11] to consider link removal "one-by-one" from the topology where the least utilized link is selected first. First of all, if link $C \rightarrow D$ is put to sleep, its load is re-routed through the alternative path $C \rightarrow A \rightarrow D$ and the resulting utilization on link $A \rightarrow D$ becomes $105 \%$. Hence, $C \rightarrow D$ cannot be put to sleep because it causes the network to become overloaded. Link $A \rightarrow D$ can be put to sleep because the alternative path $A \rightarrow B \rightarrow D$ will have MLU of 37.5\%. Therefore, the resulting MLU in the network is $60 \%$ and only one link can be put to sleep.

If the link weights are optimized as shown on the right side of Fig. 1, both links $C \rightarrow D$ and $A \rightarrow D$ can be put to sleep without causing the network to become overloaded. The traffic demands $C-D$ and $A-D$ are routed along the new paths $C \rightarrow A \rightarrow B \rightarrow D$ and $A \rightarrow B \rightarrow D$ respectively. The MLU of the resulting network becomes $52.5 \%$ even though two links are now put to sleep. The optimization of the link weights has made it possible not only to reduce the MLU from $75 \%$ to $52.5 \%$ but also allow one more link to go to sleep, achieving simultaneous improvement of both load-balancing and energy efficiency objectives.

In addition to the aforementioned simple example that illustrates the effectiveness of GLA based on one traffic matrix [11][12], the proposed scheme can be further applied to more advanced approaches which also take into account traffic dynamics, by considering simultaneously multiple traffic matrices. According to the Time-driven Link sleeping (TLS) approach [7][8], both the set of sleeping links and their sleeping time are jointly determined by taking into account the patterns of traffic demands for a given period of time. When GLA is to be applied in such scenarios, additional constraints need to be considered. Specifically, a common set of IGP link weights needs to be applied across a diverse set of traffic matrices. This requires the algorithm to be robust enough to ensure optimized load-balancing performance is achieved across different time periods.

Regarding energy savings in a network system, when a network link is configured to the sleep mode, the line cards at both ends of the link are either put to sleep if the line cards have no other active links connected to them, or they can enter a lower power mode because an interface becomes inactive. Putting a whole line card or one of its interfaces inside the router to sleep is the main source of energy savings in a backbone network because optical fiber links along with their amplifiers account for only $7 \%$ of the power budget of a network [5] with the remaining $93 \%$ energy being consumed by routers. The line cards inside an IP router typically consume around $43 \%$ of the total power of the router [6] and therefore, significant energy efficiency can be achieved by putting links into sleep mode because of the effect it has on the power consumption of line cards. 


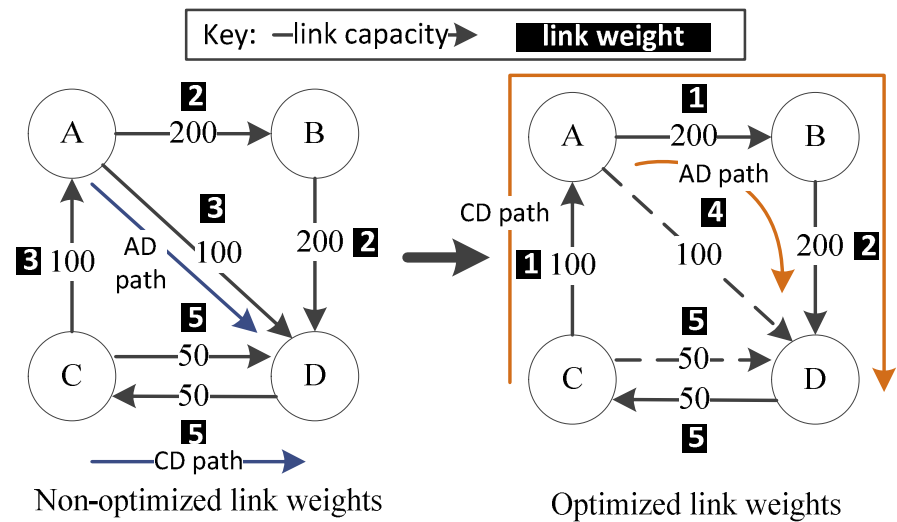

Fig. 1. Example network topology to illustrate optimization of link weights.

\section{Problem Formulation and Existing ETE Schemes}

\subsection{Problem Formulation}

Table 1. Definition of Symbols.

\begin{tabular}{|l|l|}
\hline Variable & Description \\
\hline$G(N, L)$ & Directed graph with $N$ being set of nodes and $L$ being set of links \\
\hline$U$ & Maximum link utilization \\
\hline$E$ & Energy efficiency \\
\hline$c_{i j}$ & Bandwidth capacity of link from node $i$ to node $j$ \\
\hline$t^{s d}$ & Traffic demand from node $s$ to node $d$ \\
\hline$f_{i j}^{s d}$ & Traffic demand from node $s$ to node $d$ that traverses link from node $i$ to node $j$ \\
\hline$f_{i j}$ & Total traffic demand on link from node $i$ to node $j$ \\
\hline$\alpha$ & Maximum allowable utilization of link capacity \\
\hline
\end{tabular}

The joint-optimization of load-balancing and energy efficiency in a network can be expressed with the following two objectives:

minimize $U$

maximize $E$

subject to:

$$
\begin{aligned}
& \sum_{j=1}^{|N|} f_{i j}^{s d}-\sum_{j=1}^{|N|} f_{j i}^{s d}=\left\{\begin{array}{cl}
t^{s d} & \forall s, d, i=s \\
-t^{s d} & \forall s, d, i=d \\
0 & \forall s, d, i \neq s, d
\end{array}\right. \\
& f_{i j}<\frac{\alpha}{100} \times c_{i j} \quad \forall i, j \text { with } \alpha \in[0,100]
\end{aligned}
$$

Eq. 1 represents the first objective of GLA which is the minimization of the Maximum Link Utilization (MLU) in the network in order to achieve load-balancing. For the GLA scenarios based on one traffic matrix, it refers to the MLU related to that snapshot only. For the GLA scenarios involving multiple traffic matrices (e.g. based on the TLS ETE scheme), this refers to the worst-case scenario across all considered traffic matrices. Eq. 2 represents the second objective of GLA which is the maximization of the energy saving gains given by an existing ETE scheme. Again, the definition of $E$ is specific to individual ETE schemes we consider and will be introduced in detail in Section 4.2. Eq. 3 represents the standard flow conservation constraint. Eq. 4 ensures that whenever a reduced topology is used, all active links should have their utilization below a given threshold $\alpha$, which is determined by the network operator. That is, with a set of links being put into sleep mode, the MLU should not exceed the threshold $\alpha / 100$ of the link capacity. In addition to the constraints in Eq. 3 and 4 , the 
network needs to remain fully connected when links are configured to sleep mode so that there is always a path between any two nodes in the reduced network topology.

\subsection{Existing ETE Schemes}

In this subsection, we give a brief review of the three different offline ETE schemes on which GLA is applied in this paper. The first two schemes, Least Flow (LF) and Most Power (MP), were introduced in [21] and [22] respectively. The third ETE scheme on which GLA was evaluated is Time-driven Link Sleeping (TLS) which is our own ETE scheme and was introduced in [7] and [8]. The major difference between the first two schemes and TLS is that the first two schemes operate on one single traffic matrix snapshot at a time and do not consider a collection of dynamic traffic matrices as TLS does. It is also worth mentioning that each of these three schemes has a different way of calculating Eq. 1 and 2 in Section 4.1 .

\subsubsection{The Least Flow Scheme}

In [21], the authors use an ETE scheme called Least Flow (LF). LF iteratively selects the least loaded link in the network as candidate for sleeping. The selected link can only go to sleep if the full connectivity of the network topology is maintained and the resulting MLU is below a given threshold when the link enters sleep mode. Otherwise, the next least loaded link is selected for sleeping consideration until all the links in the network have been investigated.

During the operation of GLA on top of LF, the value of Eq. 1 is the MLU value when the single traffic matrix is mapped onto the network with its full topology. The MLU value obtained is then used as the value of $\alpha$ in Eq. 4 . The energy efficiency (value of Eq. 2) of LF is calculated as shown by Eq. 5 where $|B|$ is the number of sleeping links and $|L|$ is the total number of links in the network. The complexity of the LF scheme was given as $O\left(|L| .|N|^{2} .(|L|+|N| . \log |N|)\right)$ in $[21]$.

$$
E=\frac{|B|}{|L|}
$$

\subsubsection{The Most Power Scheme}

The Most Power (MP) ETE scheme presented in [22] is similar to LF. MP iteratively selects the link which consumes the largest amount of power in the network as candidate for sleeping. The selected link can only go to sleep if the full connectivity of the network is maintained and the resulting MLU is below a given threshold when the link is sleeping. Otherwise, the next link which consumes the most power becomes candidate for removal.

During the operation of GLA on top of MF, the value of Eq. 1 is calculated in the same way as for the LF scheme above. The MLU value obtained is then used as the value of $\alpha$ in Eq. 4. The energy efficiency (value of Eq. 2) of MP is calculated as shown in Eq. 6 where $P_{l}$ is the power consumed when the link with index $l$ is active and $B$ is the set of links that are sleeping. As mentioned in Section 3, the energy savings due to a link being put into sleep mode can pre-dominantly be attributed to the line cards which are connected to the link. The complexity of the MP scheme was given as $O\left(|L| \cdot|N|^{2} \cdot(|L|+|N| \cdot \log |N|)\right)$ in [22].

$$
E=\frac{\sum_{l=0: l \in B}^{|L|} P_{l}}{\sum_{l=o}^{|L|} P_{l}}
$$

\subsubsection{The Time-driven Link Sleeping Scheme}

The main design principle of TLS [7][8] is that sometimes it is not practical to re-calculate the links to go to sleep for each traffic matrix at short-time scale due to stability requirements in the network. Since it has been observed that many operational networks ([31][32][33][34][17]) exhibit a regular diurnal traffic pattern where the traffic demands are high during the day and low during the night, TLS takes advantage of this traffic pattern by having two network configurations: "full network topology" and "reduced network topology". The "full network topology" is the network configuration where all the links in the network are active while the "reduced network topology" is the network configuration where some links are put to sleep for energy efficiency. The first configuration is used during peak time and the second one during off-peak time. The TLS scheme is responsible for jointly determining both the sleeping links set and its sleeping duration, which is effectively the duration of the off-peak time.

In the operation of GLA on top of TLS, the objective value, $U$, in Eq. 1 is equal to the worst-case MLU in the network when all traffic matrices are considered. Specifically, the metric $U$ in Eq. 1 represents the peak-time MLU in the network to be optimized. The MLU constraint for the off-peak time, represented by $\alpha$ in Eq. 4, depends on either the obtained peak-time MLU or is pre-determined by the network operator. The energy efficiency (value of Eq. 2) of TLS is calculated according to Eq. 7, where $|B|$ is the number of sleeping links in the "reduced network topology", $T_{o p}$ is the time duration during which the "reduced network topology" is operated, $|L|$ is the total number of links in the network and $T$ is the total operation time under consideration. According to Eq. 7, the energy efficiency of TLS can only be increased by increasing the nominator in Eq. 7 since the denominator is fixed. Intuitively, an increase in the number of sleeping links may lead to a smaller off-peak duration 
(i.e. sleeping time $T_{o p}$ ), because the capacity of network is reduced and only a smaller number of traffic matrices can now be supported with the reduced network topology. Therefore, a trade-off needs to be obtained between $|B|$ and $T_{o p}$. The complexity of the TLS scheme was given as $O\left(|L| . Y . K .|N|^{2} \cdot(|L|+|N| \cdot \log |N|)\right)$ in [8] where the newly introduced symbols $Y$ and $K$ are the number of traffic matrices collected in each day and the number of days considered respectively.

$$
E=\frac{|B| \times T_{o p}}{|L| \times T}
$$

\section{Green Load-balancing Scheme}

\subsection{Scheme Overview}

Since it is well-known that computing the optimal link weights for basic load-balancing alone is already an NP-hard problem [35], here we propose a new scheme, Green Load-balancing Algorithm (GLA), which is based on meta-heuristics (evolutionary/genetic algorithms) to find the optimized IGP link weights which can solve the more complicated problem of the joint-optimization of load-balancing and energy efficiency in a backbone network.

More specifically, GLA is used to solve the problem of finding the set of optimized green IGP link weights which caters for both objectives 1 and 2 as represented by Eq. 1 and 2 respectively in Section 4.1. GLA is implemented in the form of a customized version of the Non-dominant Sorting Genetic Algorithm (NSGA-II) [36]. NSGA-II operates in a similar fashion to traditional genetic algorithms. NSGA-II has been chosen because it is a multi-objective algorithm which preserves diversity and elitism of the solution space and has low computational complexity. NSGA-II can be used to find the Paretooptimal front of a solution space. This Pareto-optimal front arises due to the presence of two objectives in the problem formulation: load-balancing and energy efficiency. Intuitively, load-balancing and energy efficiency through link sleeping are two conflicting objectives. This is because load-balancing aims to reduce the load on highly-utilized links by shifting the traffic demands on these links to less utilized links in the network, while energy efficiency requires the traffic to be concentrated on a subset of active links and putting the non-utilized links to sleep, which results in the active links becoming highly-utilized. The conflict between the two objectives gives rise to a Pareto-optimal front in the sense that, when the MLU is reduced for load-balancing purposes, the energy efficiency objective will be sacrificed because of the more constrained environment for the different ETE schemes. Hence, an optimized trade-off needs to be obtained between these two objectives. In addition to the basic NSGA-II operations, two custom operators are also introduced to further enhance its performance through more efficient search in the solution space.

\subsection{Solution Encoding}

In the genetic algorithm, the solution (i.e. the set of IGP link weights in GLA) is encoded through a chromosome. A chromosome is made up of a number of genes, which is equal to the number of links in the network in our case. Therefore, each gene in the chromosome represents a link weight in the network and is restricted to an integer value in the range of 1 to 65534. This range corresponds to the range of values allowed for IGP link weights in the context of the three ETE schemes being studied. The GLA scheme reserves the link weight value of 65535 for representing links that are selected to be put into sleep mode, and hence they are not used to carry traffic demands according to the scheme. However it should be noted that, for the practical configuration of the operational network, an operator may apply any other mechanism to enforce link sleeping.

\subsection{Fitness Functions}

Each chromosome (i.e. solution candidate) has two distinct fitness functions in the NSGA-II algorithm which are represented by Eq. 1 and 2 in Section 4.1 respectively. As mentioned previously, since the three ETE schemes, described in Section 4.2, have different internal mechanisms, their fitness functions differ in the way they calculate Eq. 1 and 2 as described in Section 4.2 .

\subsection{Sleeping Link Crossover Operator}

A crossover operation in a genetic algorithm involves taking two chromosomes (i.e. two solutions) in the current population (set of solutions) and swapping their genes (i.e. link weights) with each other to produce two new offspring chromosomes. The aim is to produce newly generated solution candidates with better fitness values. In order to explore the solution space more efficiently and achieve quicker convergence, a customized crossover operator is designed in addition to the standard crossover operators such as two-segment crossover. This new crossover operator has been designed so that one 
parent chromosome can replace its gene with its counterpart gene in the other parent chromosome if the counterpart gene represents a sleeping link.

Fig. 2 shows the operation of the new crossover operator. A chromosome in this operation is made up of two rows. The top row contains the IGP link weights while the bottom one contains a binary array which indicates whether a particular link is active or not. A " 1 " value means that a link is active and " 0 " means the link is sleeping. The IGP link weight of an active link in a chromosome is changed with its counterpart in the other parent chromosome only if its counterpart is marked as sleeping. The arrows between the two parent chromosomes in Fig. 2 show when link weight change occurs and the direction of the change. This operation is similar to "XORing" the bottom row of the two parent chromosomes. This new crossover operator allows "good" link weights which promote link sleeping to propagate through the population.

\subsection{Link Utilization Mutation Operator}

A mutation operation in a genetic algorithm involves taking one chromosome (i.e. solution candidate) in the population and modifying one or more genes (i.e. link weights) in the chromosomes. This operation is done so that new genes, which do not exist in the current population, are introduced in the population with the aim that this will increase the fitness value of the selected chromosomes. A new mutation operator has been developed based on the percentage utilization of each link when a traffic matrix is mapped onto the topology. In this new mutation operator, each link weight has $P_{u m u t}$ probability of mutating which is equal to the utilization percentage of that link divided by the MLU in the network. As such, highly utilized links will have a higher probability of mutating compared to links with low utilization. The probability that a chromosome in the parent population will begin this utilization-based mutation is given by $P_{m u t} / 2$ where $P_{m u t}$ is the probability of a chromosome undergoing mutation and there is $P_{m u l} / 2$ probability that the chromosome will undergo the other standard mutation operators such as random gene mutation.

If a link is selected to be mutated, its link weight will be increased according to Eq. 8 where $W_{n}$ is the new link weight. $W_{o}$ is the old link weight and is used as the mean of a normal distribution $V$ with standard deviation $\varepsilon W_{o}$ where $\varepsilon$ is a fractional multiplier lower than 1. The rationale behind Eq. 8 is that highly utilized links will have their link weights increased, and hence become less likely to be chosen by IGP for routing traffic demands. This is likely to cause the load of these links to decrease if alternative shorter paths are identified to route the traffic demands.

$$
W_{n}=W_{o}+\left|W_{o}-V\left(W_{o}, \varepsilon W_{o}\right)\right|
$$

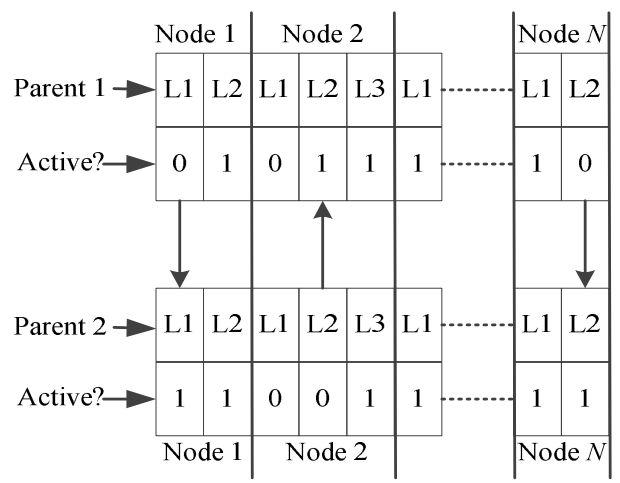

Fig. 2. Sleeping link crossover operator.

\subsection{Overall Operation of GLA}

At the beginning of GLA, an initial population of random chromosomes is generated. A chromosome consisting of the default link weights is also added to the initial population. An offspring population is then created through the joint application of crossover and mutation operators on some randomly selected chromosomes in the parent population. In addition to the traditional crossover and mutation operators such as two-segment crossover and random gene mutation, the two custom crossover and mutation operators, described in the previous two subsections, are applied. These customized genetic operators help the exploration of the search space in a more efficient manner. The parent and offspring populations are then merged and sorted according to the fitness and diversity scores of the individual chromosomes to create a new parent population. The algorithm stops when a given target number of generations has been calculated and/or there has been no improvement in the obtained solutions since a given number of generations. The problem-independent complexity of one iteration of NSGA-II is reported to be $O\left(M X^{2}\right)$ [36] where $M$ is the number of objectives in the problem (i.e. two in our 
case) and $X$ is the population size. The complexity of the evaluation of the fitness functions for each solution in the population depends on the ETE schemes used and has been reported in Section 4.2.

\subsection{Implementation of GLA in Operational Networks}

For the original LF and MP ETE schemes, the authors propose to broadcast to routers the links that need to be put to sleep for each traffic matrix [21][22]. After the routers receive the notification of which links to put to sleep and put the links to sleep, all the routers in the network need to recalculate their forwarding tables to reflect the new connectivity of the network. There may be some instability during this recalculation of forwarding tables, such as forwarding loops. When GLA is applied to the LF and MP ETE schemes, the only implementation change is that routers will receive the new link weights that need to be assigned to the remaining active links as well as the links to be put to sleep, as done previously. All the routers will recalculate the forwarding tables as done previously, albeit with new link weights. The use of new link weights does not fundamentally introduce additional network instabilities, as the process of recalculating the forwarding tables without some links is similar to doing the recalculation with new link weights; since the relationship between the links has changed in both cases and all routers always need to do a complete recalculation of the forwarding tables.

As mentioned previously in Section 4.2.3, the TLS scheme uses only two network configurations which are implemented in the form of two forwarding tables to represent the peak and off-peak network topologies. Packets are marked by the source routers in order to select which forwarding table the intermediate routers will use to route the packets towards their destination. For both the plain TLS and TLS with GLA, the forwarding tables are calculated in an offline fashion and even though TLS with GLA uses new link weights, in addition to which links to put to sleep, to calculate the off-peak forwarding table, the routers will only see an additional off-peak forwarding table as they would with the plain TLS ETE scheme. Hence, it can be concluded that plain TLS and TLS with GLA have a similar effect on the network. The only actual instability that plain TLS and TLS with GLA introduce in the network is when the routers switch between the forwarding tables. This happens only twice per day compared to every 15 minutes for the LF and MP ETE schemes and therefore TLS can be considered substantially more stable than these schemes.

\section{Performance Evaluation}

\subsection{Network Scenario}

We evaluate the performance of GLA on top of the three different ETE schemes, LF [21], MP [22] and TLS [7][8], by using the operational network topology, GÉANT and its published traffic matrices [37]. GÉANT is a European academic network which has allowed researchers access to its network topology and traffic matrices. The published topology consists of 23 Points-of-Presence (PoPs) and 74 unidirectional links of varying bandwidth capacities which are described in Table 2 below. The total power consumed by active links is also given in the table. These values were calculated from the power consumption model of line cards in [38] with the assumption that line cards are responsible for most of the energy consumption of a link [5][6]. The power values are used by the $2^{\text {nd }}$ ETE scheme, MP, to decide which link to put to sleep first. Out of the three ETE schemes evaluated in this paper, MP is the only ETE scheme which was originally designed to make use of different power values for links of different capacity while the other two ETE schemes, namely LF and TLS, assumed that the power consumed by each link is the same. In order to fairly evaluate GLA, the original method by which the energy savings are calculated for each scheme is kept in this paper and has been described in detail in Section 4.2.

TLS operates on a collection of traffic matrices by nature and therefore, we consider 480 consecutive traffic matrices at 15-minute intervals from Monday midday to Saturday midday according to the historical GÉANT traffic matrix data set [37]. The statistical characteristics of the traffic matrices during this period are given in Table 3. The LF and MP ETE schemes only focus on each standalone traffic matrix. For these schemes, we choose 10 traffic matrices from the set of traffic matrices used to evaluate TLS. The 10 traffic matrices were chosen by taking 2 traffic matrices from each subset of traffic matrices which has an MLU close to the Maximum, Minimum, Mean and $1^{\text {st }}$ and $3^{\text {rd }}$ Quartiles MLU as specified in Table 3.

Table 2. Power used due to an active link

\begin{tabular}{|c|c|c|c|c|}
\hline & Number of Links, L & Bandwidth (Mbps) & $\begin{array}{l}\text { Power (W) / } \\
\text { link }\end{array}$ & $L \times P(W)$ \\
\hline & 32 & 9953 & 1120 & 35840 \\
\hline & 2 & 4876 & 560 & 1120 \\
\hline & 32 & 2488 & 280 & 8960 \\
\hline & 8 & 155.2 & 98 & 784 \\
\hline Total & 74 & & & 46704 \\
\hline
\end{tabular}




\begin{tabular}{|l|l|}
\hline Maximum Link Utilization & Value (\%) \\
\hline Maximum & 90.9 \\
\hline Minimum & 30.9 \\
\hline Mean & 58.6 \\
\hline $1^{\text {st }}$ Quartile & 44.5 \\
\hline $2^{\text {nd }}$ Quartile & 55.9 \\
\hline $3^{\text {rd }}$ Quartile & 74.2 \\
\hline
\end{tabular}

There are three different sets of link weights which are compared in this paper: Default, Interior Gateway Protocol Weight Optimizer $(I G P-W O)$ and GLA. The Default link weights are the actual link weights applied in practice. IGP-WO contains link weights which are optimized following [35] for general load-balancing purpose only, without any energy awareness. These two link weight setting strategies are used as benchmarks to evaluate the energy saving gains obtained by GLA applied on common ETE schemes.

Both GLA and IGP-WO are run with 10 different seeds to get the average performance as well as the $95 \%$ confidence interval performance since they are both based on meta-heuristics and therefore are not deterministic in their behavior. The number of random and independent runs of GLA and IGP-WO to be done was chosen to be ten because the designers of NSGA-II used this number to evaluate and compare NSGA-II with other competing algorithms [36]. It should be noted that Default is a deterministic algorithm and therefore, its performance does not vary between different runs. As mentioned previously, GLA produces a set of Pareto-optimal solutions for each seed. Each solution candidate shows a different trade-off between energy-savings and load-balancing. As we aim to achieve energy efficiency without substantially sacrificing conventional traffic engineering (i.e. load balancing) performance, we decided to exclude all solutions which have an MLU which is $\Psi \%$ (e.g. 3\% in our evaluation scenarios) above the lowest MLU given by the different sets of GLA link weights. For each seed, the best solution is then chosen by identifying the solution among the remaining solution candidates which has the lowest ratio of MLU to energy efficiency. TLS was further evaluated by having different MLU constraints, represented by $\alpha$ in Eq. 4, during off-peak time where the "reduced network topology" is applied. The different off-peak MLU constraints represent the different degrees of conservativeness by the network operator during off-peak time.

\subsection{Simulation Results}

Table 4 demonstrates the performance of the three sets of link weights for the LF and MP ETE schemes specified in Section 4.2. The performance is measured in terms of the decrease in Maximum Link Utilization, $-\Delta U$, and increase in energy efficiency, $\Delta E$, when compared to the results given by the Default link weights. The Maximum Link Utilization given by a set of link weights for the MP and LF ETE scheme is calculated by averaging the utilization of the most utilized link in the network for the 10 traffic matrices that were considered as description in Section 6.1. On average, the number of sleeping links computed by LF has increased by $15.8 \%$ while the MLU has decreased by $30.6 \%$ when the GLA link weights were used instead of the Default ones. This shows that GLA can reduce the MLU while still achieving significantly higher energy efficiency. In contrast, the IGP-WO link weights were only able to achieve a small improvement in the energy efficiency after the weights have reduced the MLU in the network. This is shown by the negative sign for $\Delta E$. It is observed that GLA performs slightly better than IGP-WO in terms of load-balancing even though GLA considers energy-savings at the same time. For MP, the average amount of energy saved by GLA has increased by $1.11 \%$ while reducing the MLU by $30.9 \%$ compared to the results given by the Default link weights. When IGP-WO link weights are used, it was not possible to improve the energy efficiency when the MLU of the network is reduced. Table 4 also includes the $95 \%$ confidence interval $(95 \% \mathrm{CI})$ values for the energy efficiency and maximum link utilization so as to illustrate the statistical accuracy of our results (since the confidence intervals are symmetrical around the average value, for simplicity we are showing only the right bound of the confidence interval). It can be seen that the 95\% CI values follow the same trend as the average values which confirms that GLA achieves better energy efficiency and load-balancing compared IGP-WO.

Table 4. Performance comparison of the three sets of link weights for LF and MP

\begin{tabular}{|l|l|l|l|l|l|l|l|l|}
\hline \multirow{2}{*}{$\begin{array}{l}\text { ETE } \\
\text { Scheme }\end{array}$} & \multicolumn{3}{|c|}{ IGP-WO } & \multicolumn{4}{c|}{ GLA } \\
\cline { 2 - 9 } & \multicolumn{2}{|c|}{$-\boldsymbol{\Delta U}(\boldsymbol{\%})$} & \multicolumn{2}{c|}{$\boldsymbol{\Delta E}(\boldsymbol{\%})$} & \multicolumn{2}{c|}{$-\boldsymbol{\Delta U}(\boldsymbol{\%})$} & \multicolumn{2}{c|}{$\boldsymbol{\Delta E}(\boldsymbol{\%})$} \\
\cline { 2 - 9 } & $\mathbf{9 5 \%} \mathbf{C I}$ & Avg. & $\mathbf{9 5 \%} \mathbf{C I}$ & Avg. & $\mathbf{9 5 \%}$ CI & Avg. & $\mathbf{9 5 \%}$ CI & Avg. \\
\hline LF & 29.6 & 26.8 & 5.83 & 1.09 & 31.4 & 30.6 & 17.8 & 15.8 \\
\hline MP & 29.1 & 26.3 & -6.30 & -11.3 & 31.6 & 30.9 & 2.85 & 1.11 \\
\hline
\end{tabular}


Regarding TLS, Fig. 3 shows that GLA can achieve a substantial average improvement in energy efficiency (the energyefficiency of TLS is calculated by using Eq. 7 in Section 4.2.3) of 238\% and 144\% compared with Default and IGP-WO link weights respectively when $\alpha$ (in Eq. 4) is set equal to the worst-case MLU given by the "full network topology", $U$. Effectively, $\alpha$ represents the worst-case MLU that can be observed during the entire off-peak operation duration of the "reduced network topology". Similar observation is obtained when $\alpha$ is further reduced to $65 \%$ and $60 \%$ respectively. The energy efficiency decreases when $\alpha$ is decreased because of the more conservative constraint for TLS. Table 5 shows that the high energy efficiency obtained using GLA is not at the expense of load-balancing since the GLA values for load-balancing are lower than those for IGP-WO. Similar to Table 4, Table 5 also includes the 95\% CI values for the energy efficiency and maximum link utilization and they follow the same trend as the average values. That is when the value of $\alpha$ is reduced, the energy efficiency given by TLS also is also reduced. The 95\% CI values are represented in the graph of Fig. 3 by the statistical bars.

Fig. 4 shows the actual MLU performances across the 5 days when Default, IGP-WO and GLA link weights are applied to the network. It is interesting to see that when $\alpha$ is set equal to $U$, the off-peak duration of GLA is even able to cover the entire 5-day period because the difference between the peak and off-peak MLU under GLA is zero (also see Table 5). It is acceptable for a network to not use some links at all because this will reduce the operational costs even if the network operator has already invested capital in the network. The network operator can always put the always-sleeping links back on when there is a need for extra capacity in the network. When $\alpha$ is set below $U$, link sleeping can be only configured within a specific period on daily basis. This is shown by the dark areas for the Default link weights in Fig. 4 and for the GLA link weights in Fig. 5 where $\alpha$ is set equal to $60 \%$.

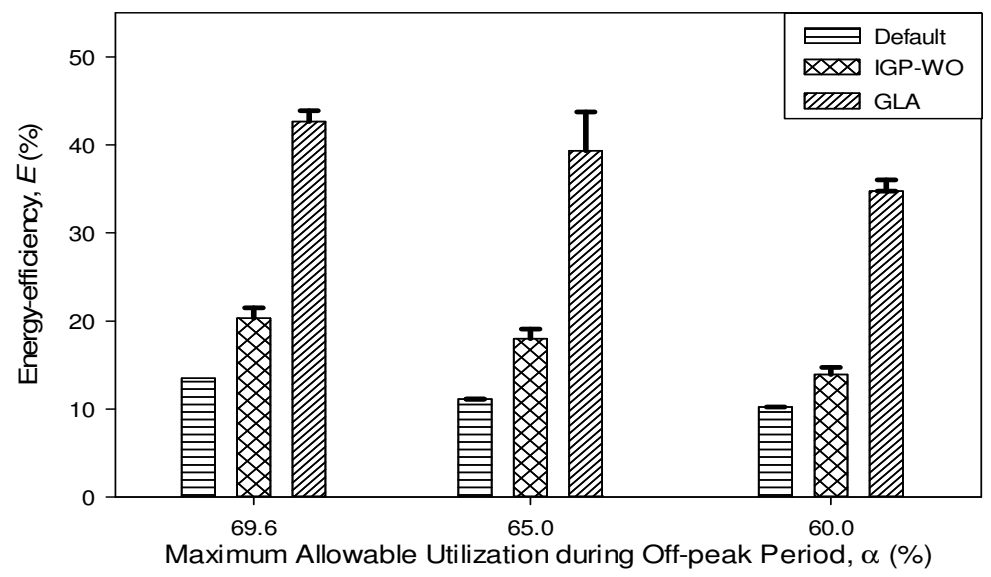

Fig. 3. Energy efficiency of TLS using the different sets of link weights.

Table 5. Performance of GLA for the three sets of link weights for TLS

\begin{tabular}{|c|c|c|c|c|c|c|c|c|c|c|}
\hline \multirow{3}{*}{$\begin{array}{c}\alpha \\
(\%)\end{array}$} & \multicolumn{2}{|c|}{ Default } & \multicolumn{4}{|c|}{ IGP-WO } & \multicolumn{4}{|c|}{ GLA } \\
\hline & \multirow[t]{2}{*}{$U(\%)$} & \multirow[t]{2}{*}{$E(\%)$} & \multicolumn{2}{|c|}{$U(\%)$} & \multicolumn{2}{|c|}{$E(\%)$} & \multicolumn{2}{|c|}{$U(\%)$} & \multicolumn{2}{|c|}{$E(\%)$} \\
\hline & & & $95 \%$ CI & Avg. & $95 \% \mathrm{CI}$ & Avg. & $95 \%$ CI & Avg. & $95 \%$ CI & Avg. \\
\hline 69.7 & 90.9 & 13.5 & 70.1 & 70.1 & 21.3 & 20.3 & 69.9 & 69.7 & 43.7 & 42.7 \\
\hline 65.0 & 90.9 & 11.1 & 70.1 & 70.1 & 18.9 & 18.0 & 69.7 & 69.6 & 42.9 & 39.3 \\
\hline 60.0 & 90.9 & 10.2 & 70.1 & 70.1 & 14.6 & 13.9 & 69.7 & 69.6 & 35.8 & 34.7 \\
\hline
\end{tabular}




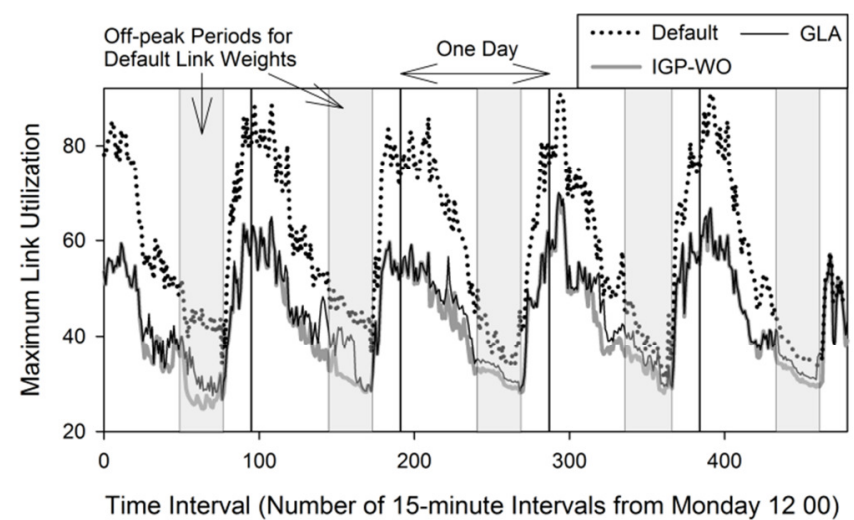

Fig. 4. The MLU variation across 5 days for Default, IGP-WO and GLA link weights when $\alpha$ is set equal to $U$.

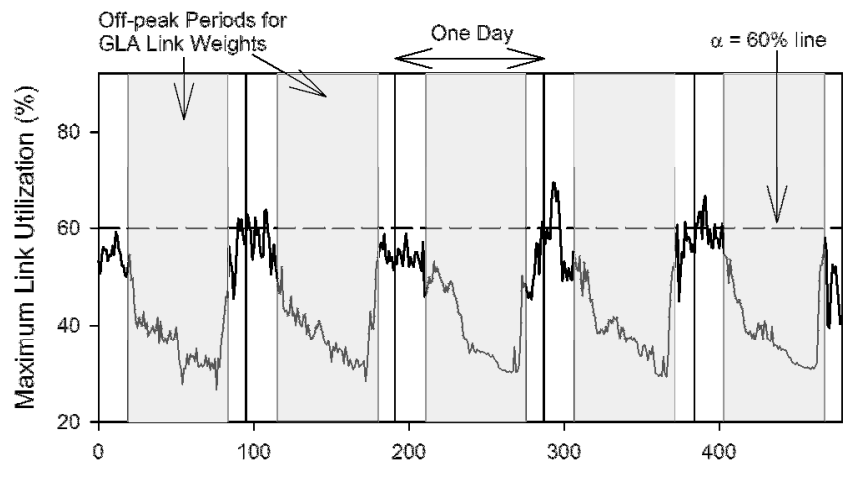

Fig. 5. The MLU variation across 5 days for GLA link weights when $\alpha$ is set equal to $60 \%$, which is below $U$.

\section{Enhanced GLA for TLS}

\subsection{Solution-enhancement Heuristic}

The performance of GLA for TLS can be improved further if GLA is further customized for TLS through the use a Solution-enhancement Heuristic (SH). This enhanced version of GLA is called Green Load-balancing Algorithm with Solution-enhancement Heuristic (GLA-SH). SH operates on the best solution in the population at the end of each iteration of GLA. The best solution is determined by a single aggregated objective function represented by the ratio of Maximum Link Utilization to energy efficiency as given by Eq. 1 and 2 in Section 4.1 respectively. Fig. 6 shows the operation flowchart of $\mathrm{SH}$. The chromosome (i.e. solution candidate) selected for improvement is first evaluated to find the size of the off-peak duration. In TLS, the energy efficiency is effectively represented by the number of sleeping links multiplied by the off-peak duration.

If the length of the off-peak duration is at its maximum, the duration cannot be further increased to improve the energy efficiency. The only option is to increase the number of sleeping links instead. This involves diverting load from certain active links in order to enable them to sleep. This diversion of load is done through the increase of the IGP link weights of certain links. SH first identifies the traffic matrix which gives the worst-case MLU when its traffic demands are routed with the off-peak "reduced network topology". The next step involves the creation of a list of active links ranked in ascending order according to the utilization of the links. The first link in the list is the least utilized in the network and therefore, it is easier to shift its load to other links. This link will be first chosen to have its link weight increased. After each link weight increase, the modified chromosome is re-evaluated to see if the energy efficiency has improved and the worst-case MLU has remained the same or has been reduced. If these criteria are met, SH stops and returns the improved chromosome to the population where it will replace the currently worst chromosome. In the case of the criteria not being met, the link weight of last modified link is restored to its original value and the next link in the list undergoes link weight increase. This process continues until either all the links in the list have been tested or improvement of the chromosome has been successful.

If the size of the off-peak duration is not at its maximum, the energy efficiency can be improved through the increase of the length of the duration of the off-peak period. The traffic matrices at the edges of the off-peak periods are first evaluated according to the off-peak "reduced network topology" to see which one gives the worst-case MLU. The identified traffic matrix is the one which has most likely caused the off-peak duration to be small because the maximum allowable utilization of any link in the network has been reached. The traffic demands of the identified traffic matrix are then routed and a list of 
active links is created. This time, the list is sorted in descending order according to utilization percentage. This is because the objective is to check whether the MLU can be reduced to allow the off-peak duration to increase for more energy efficiency gains. This is achieved by shifting load from the most utilized link by increasing its link weight. The same iterative process of link weight increase is then performed in the same manner as in the previous case. Chromosomes which cannot be improved by $\mathrm{SH}$ are tracked so that $\mathrm{SH}$ does not run on them again and the next best chromosome is used as candidate for improvement by SH. The complexity of SH is $O\left(|L| \cdot|N|^{2} \cdot(|L|+|N| \cdot \log |N|)\right)$ with SH running only once for each iteration of GLA-SH.

It is worth noting that it is possible to introduce solution enhancement heuristics for other ETE algorithms as well. These solution enhancement heuristics need to be specifically designed by taking into account their own working mechanisms in question. The development of such heuristics is however outside the scope of this paper.

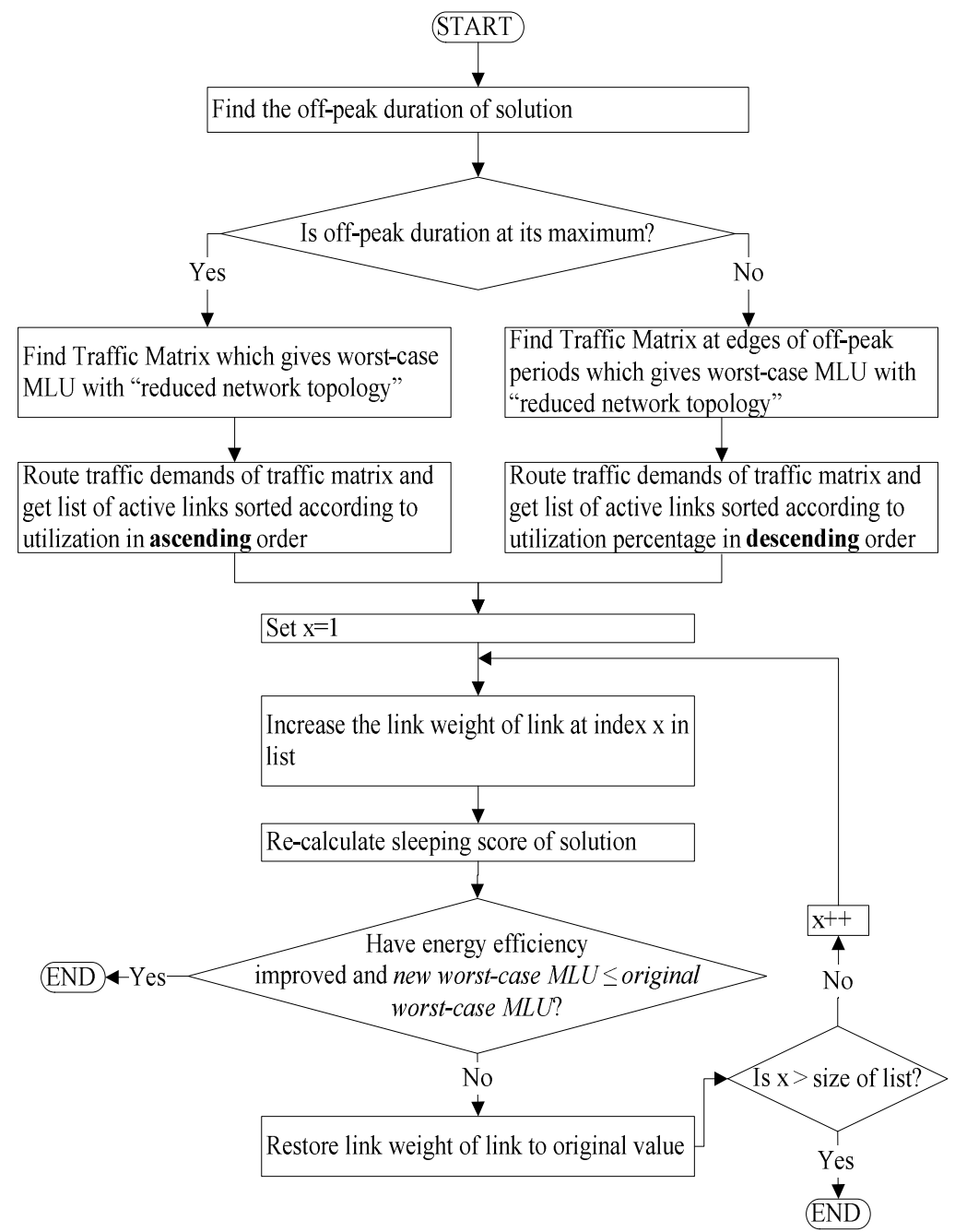

Fig. 6. Flowchart describing the operation of the Solution-enhancement Heuristic.

\subsection{Evaluation}

The GLA-SH was evaluated using the same network scenario as described in Section 6.1. The results from Table 6 and Fig. 7 show that GLA-SH can on average increase the energy efficiency of TLS by 7\% compared to plain GLA when $\alpha$ is set equal to $U$. The average improvement when $\alpha$ is set to $65 \%$ and $60 \%$ is $9.41 \%$ and $18.2 \%$ respectively. It can also be observed that the changes in maximum link utilization between GLA and GLA-SH are negligible. This behaviour of GLA$\mathrm{SH}$ is a direct result of the preference given to load-balancing over energy efficiency in our experiments by setting the value of $\Psi \%$ to a small value, i.e. $3 \%$ in our evaluation scenario. These results also show that the Solution-enhancement Heuristic can indeed improve the energy efficiency of TLS by allowing the GLA-SH scheme to escape a region of local optimality through the customization of the operation of GLA for TLS. 


\section{Packet Delay Performance of the existing ETE Schemes}

The end-to-end Maximum Packet Delay (MPD) of operational networks has become increasingly important due the popularity of real-time applications such as Voice-over-IP (VoIP) and video streaming [39]. These applications normally require a bounded MPD in order to provide end-users with good Quality-of-Experience (QoE). The three ETE schemes, described in Section 4.2: LF, MP and TLS, are oblivious to packet delay and therefore, the packet delay may substantially increase when links are put to sleep for energy saving. In this section, we present a comprehensive analysis of the MPD performance of the three different ETE schemes on the three different sets of link weights: Default, IGP-WO and GLA.

Table 7 shows the maximum, average and minimum increase in MPD when the LF ETE scheme is applied for the Default, IGP-WO and GLA link weights. The general observation is that Default link weights perform the best in terms of MPD performance while the GLA link weights scenario has the worst result. There are two main reasons behind this observation: first, the Default link weights have more freedom in their routing because they do not optimize load-balancing as GLA does. During load-balancing, the GLA and IGP-WO schemes will divert some traffic away from their original shortest paths to longer ones so as to avoid bottleneck links and therefore result in larger MPD values. The second reason is that GLA puts more links to sleep compared to the Default link weights as shown in Table 4 in Section 6.2 where the LF ETE scheme measures directly the number of links put to sleep (see Eq. 5). This leads to the network topology being less connected, forcing packets to take longer paths to reach their final destinations. GLA has also worse MPD performance compared to IGP-WO because even though the load-balancing performance is similar, more links are put to sleep by GLA compared to IGP-WO. The IGP-WO and GLA columns in Table 7 indicate that there can be large variations in MPD performance given different traffic matrices. This is due to the fact that the LF ETE scheme is putting links to sleep with the only constraints of maintaining full network connectivity and not violating the MLU threshold and therefore the LF ETE scheme does not consider any packet delay requirement. Table 8 further shows the same general trend in MPD performance for the MP ETE scheme as for the LF ETE scheme based on the three sets of link weights: Default, IGP-WO and GLA. The reasons for this observation are the same as for the LF ETE scheme.

Table 6. Comparison between GLA and GLA-SH

\begin{tabular}{|c|l|l|l|l|l|l|l|l|}
\hline \multirow{2}{*}{\begin{tabular}{c}
$\boldsymbol{\alpha}(\boldsymbol{\%})$ \\
\cline { 2 - 9 }
\end{tabular}} & \multicolumn{4}{|c|}{$\boldsymbol{U}(\boldsymbol{\%})$} & \multicolumn{3}{c|}{$\boldsymbol{E}(\boldsymbol{\%})$} & \multicolumn{2}{c|}{$\boldsymbol{U}(\boldsymbol{\%})$} & \multicolumn{2}{c|}{$\boldsymbol{E}(\%)$} \\
\cline { 2 - 9 } & $\mathbf{9 5 \%} \mathbf{C I}$ & Avg. & $\mathbf{9 5 \%}$ CI & Avg. & $\mathbf{9 5 \%}$ CI & Avg. & $\mathbf{9 5 \%}$ CI & Avg. \\
\hline 69.6 & 69.9 & 69.7 & 43.7 & 42.7 & 69.8 & 69.6 & 47.0 & 45.7 \\
\hline 65.0 & 69.7 & 69.6 & 42.9 & 39.3 & 69.6 & 69.5 & 45.0 & 43.0 \\
\hline 60.0 & 69.7 & 69.6 & 35.8 & 34.7 & 69.7 & 69.5 & 43.6 & 41.0 \\
\hline
\end{tabular}

Table 7. Packet delay increase for the reduced network topology due to the Default, IGP-WO and GLA link weights for LF ETE scheme

\begin{tabular}{|l|l|l|l|}
\hline \multirow{2}{*}{$\begin{array}{c}\text { Increase in } \\
\text { Maximum Packet Delay }\end{array}$} & \multicolumn{3}{|c|}{ Value (\%) } \\
\cline { 2 - 4 } & Default & IGP-WO & GLA \\
\hline Maximum & 2.56 & 80.0 & 81.5 \\
\hline Average & 0.627 & 19.8 & 44.2 \\
\hline Minimum & 0.149 & 0.635 & 2.58 \\
\hline
\end{tabular}

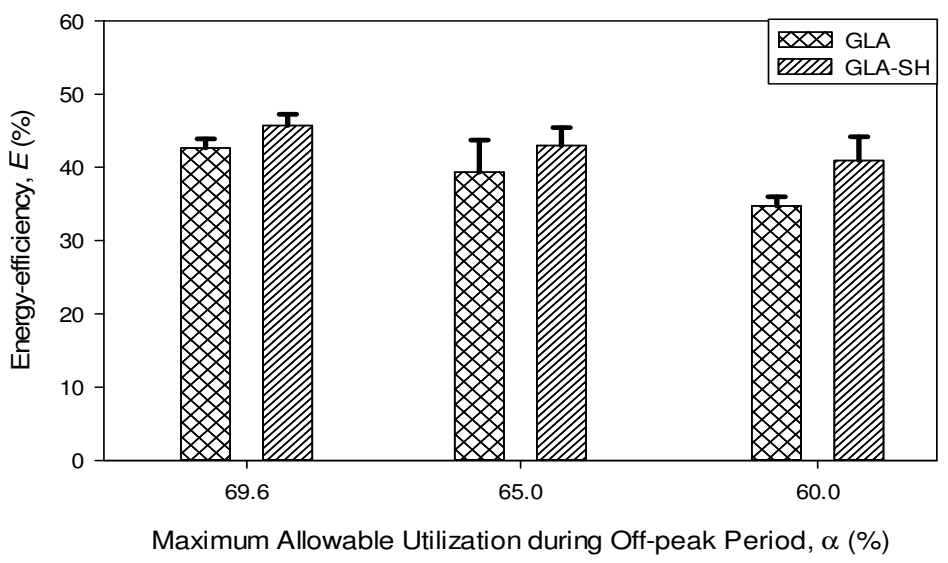


Fig. 7. Comparison of energy efficiency between GLA and GLA-SH.

Table 8. Packet delay increase for the reduced network topology due to the Default, IGP-WO and GLA link weights for MP ETE scheme

\begin{tabular}{|l|l|l|l|}
\hline \multirow{2}{*}{$\begin{array}{c}\text { Increase in } \\
\text { Maximum Packet Delay }\end{array}$} & \multicolumn{3}{|c|}{ Value (\%) } \\
\cline { 2 - 4 } & Default & IGP-WO & GLA \\
\hline Maximum & 25.39 & 88.0 & 83.6 \\
\hline Average & 6.99 & 55.3 & 78.9 \\
\hline Minimum & 2.97 & 0.713 & 55.8 \\
\hline
\end{tabular}

As mentioned in Section 4.2.3, TLS uses a full and a reduced network topology for peak and off-peak time operation respectively. Table 9 shows the MPD performance of TLS when the Default link weights are used. Upon analysis of the reduced network topologies which were generated with the Default link weights and various values of $\alpha$, it was found that all of them are the same and have 33 sleeping links each. Since the reduced network topology for the various values of $\alpha$ is the same (but have different off-peak durations), the value of the maximum increase in MPD is the same. Another observation is that the maximum MPD values for the reduced network topologies are smaller compared to the values in Table 7. Further analysis of the number of sleeping links in each ETE scheme shows that on average, 39.6 links are sleeping in the LF ETE scheme compared to 33 links for TLS ETE scheme. This larger number of sleeping links reduces the connectivity of the network, which explains the increase of the MPD. TLS has a lower number of sleeping links because it trades off sleeping links for a longer off-peak duration to maximize energy savings with only two network topologies. Table 9 also shows the maximum increase in MPD when IGP-WO link weights are used for both the full and reduced TLS network topologies. The main observation is that the MPD is very large. As mentioned previously, the IGP-WO algorithm is oblivious to packet delay and optimizes the load-balancing performance only. Table 9 shows GLA-SH link weights have the worst MPD performance among the three sets of link weights. This is because GLA-SH puts a significantly larger number of links to sleep and achieves a better load-balancing performance compared to the other scenarios as shown in Table 5 and 6. As mentioned in Section 6.2, TLS uses only a single reduced network topology all the time when $\alpha$ is set to 69.7 for the GLA-SH link weights and therefore, the MPD performance remains the same during both peak and off-peak time. For values of $\alpha$ of 65 and 60, two network topologies are used for peak and off-peak operation. The reduced network topology used during off-peak time has some links put to sleep compared to the full network topology which has no sleeping links. As a result, there is a higher probability for the MPD to increase when the reduced topology is used.

Table 9. Maximum packet delay increase due to the Default, IGP-WO and GLA link weights for TLS ETE scheme

\begin{tabular}{|c|c|c|c|c|c|c|}
\hline \multirow{3}{*}{$\boldsymbol{\alpha}$} & \multicolumn{6}{|c|}{ Maximum increase in Maximum Packet Delay (\%) } \\
\cline { 2 - 7 } & \multicolumn{2}{|c|}{ Default } & \multicolumn{2}{c|}{ IGP-WO } & \multicolumn{2}{c|}{ GLA-SH } \\
\cline { 2 - 7 } & Full NT & Reduced NT & Full NT & Reduced NT & Full NT & Reduced NT \\
\hline 69.6 & 0 & 0.336 & 54.1 & 54.1 & 77.3 & 77.3 \\
\hline 65.0 & 0 & 0.336 & 54.1 & 54.1 & 77.0 & 80.0 \\
\hline 60.0 & 0 & 0.336 & 54.1 & 54.1 & 77.0 & 80.0 \\
\hline
\end{tabular}

\section{Improvement to current ETE Schemes for Maximum Packet Delay Constraint}

In the previous section, it was indicated that the MPD performance of the three plain ETE schemes can vary substantially with the three sets of link weights. The current ETE schemes do not have a deterministic way of ensuring that a bounded MPD performance is maintained. Therefore, there is no guarantee that the MPD performance will remain within the tolerance limits of network operators. In light of this observation, it is desired to further extend the current ETE schemes so as to guarantee a minimum MPD performance while maintaining the load-balancing and energy-savings performance of the schemes. To take into account this new requirement, the MPD constraint in Eq. 8 is added to the problem formulation in Section 4.1 where $D_{\text {new }}$ is the MPD of the new network topology after it has been modified for load-balancing and/or energy-savings, $D_{\text {original }}$ is the MPD of the original network topology and $\beta$ is the maximum allowed increase ratio in MDP as defined by the network operator. The new MPD-aware ETE schemes have the same big $O$ complexity as their plain counterparts since the MPD of a reduced network topology can be calculated during the calculation of the forwarding tables.

$$
D_{\text {new }} \leq \beta \times D_{\text {original }} \quad \text { with } \beta \geq 1.0
$$


In order to enforce the new MPD constraint in Eq. 8, the way through which the three ETE schemes put links to sleep needs to be modified. The modification is that in addition to checking if the MLU and full connectivity constraints are maintained when each candidate link is considered to be put to sleep, the MPD performance of the resulting topology also needs to be examined. If the resulting MPD is higher than the upper bound, the link under consideration should not be put to sleep. The plain ETE schemes, LF, MP and TLS, which are modified to include the MPD constraint, are named LF-D, MP$\mathrm{D}$ and TLS-D respectively.

\section{Performance Evaluation of ETE Schemes with Maximum Packet Delay Constraint}

We evaluate the three new MPD-aware ETE schemes, namely LF-D, MP-D and TLS-D, by using the same network scenario as described in Section 6.1 and compare the load-balancing and energy-savings performance when the Default and GLA link weights are used. The IGP-WO link weights were not used during the evaluation because they give an MPD value which is very high. Specifically, the newly introduced extra delay due to the optimization of the link weights for loadbalancing only is substantially higher even if no links are put to sleep as shown in Table 10.

\subsection{Performance Comparison between the Original and the MPD-aware ETE Schemes}

In this section, the change in load-balancing and energy-saving performance is compared between the original and the MPD-aware ETE schemes. The chosen value of $\beta$ is 1 for all the experiments described in this section. Table 11 shows that the energy-saving performance decreases when the LF-D and MP-D ETE schemes are applied to the Default link weights when compared to the original schemes. This decrease in performance is due to a smaller number of links being put to sleep, so that the network topology remains sufficiently connected to keep the MPD within the set constraint. Table 12 shows a decrease in both the energy-savings and load-balancing performance when GLA is used with the LF-D scheme. These reductions are due to the new constraint on the MPD which reduces the diversity of paths available in the network after link sleeping. A similar observation can also be made for the MP-D scheme. Table 13 shows a decrease in the energy-savings performance of the GLA-SH link weights for the TLS-D ETE scheme while the load-balancing performance remains almost the same. During the evaluation of the new MPD-aware ETE schemes, it can be observed that the energy efficiency performance of the schemes deteriorates to a greater extent compared to their load balancing performance because load balancing was prioritized over energy efficiency in the Pareto-optimal solutions of NSGA.

Table 10. Increase in MPD for the full network topology due to the IGP-WO link weights compared to the Default ones

\begin{tabular}{|l|l|}
\hline Increase in MPD & Value (\%) \\
\hline Maximum & 54.1 \\
\hline Average & 53.8 \\
\hline Minimum & 53.0 \\
\hline
\end{tabular}

Table 11. Change in energy savings when LF-D and MP-D, rather than LF and MP, are applied to the Default link weights

\begin{tabular}{|l|l|}
\hline ETE Scheme & $\Delta \boldsymbol{E}(\%)$ \\
\hline LF-D & -3.52 \\
\hline MP-D & -2.52 \\
\hline
\end{tabular}

Table 12. Change in the 95\% CI and average load-balancing and energy savings performance when LF-D and MP-D, rather than LF and MP, are applied to the GLA link weights

\begin{tabular}{|l|l|l|l|l|}
\hline \multirow{2}{*}{ ETE Scheme } & \multicolumn{2}{|c|}{$\Delta \Delta \boldsymbol{U}(\%)$} & \multicolumn{2}{c|}{$\Delta \Delta \boldsymbol{E}(\%)$} \\
\cline { 2 - 5 } & $\mathbf{9 5 \%} \mathbf{C I}$ & Avg. & $\mathbf{9 5 \%}$ CI & Avg. \\
\hline LF-D & 0.154 & -0.083 & -7.82 & -12.0 \\
\hline MP-D & -1.05 & -2.70 & -187 & -120 \\
\hline
\end{tabular}

Table 13. Change in the 95\% CI and average load-balancing and energy savings performance when TLS-D, rather than TLS, is applied to the GLA-SH link weights 


\begin{tabular}{|c|l|l|l|l|}
\hline \multirow{2}{*}{$\begin{array}{c}\alpha \\
(\%)\end{array}$} & \multicolumn{4}{|c|}{ GLA-SH } \\
\cline { 2 - 5 } & \multicolumn{2}{|c|}{$\Delta \boldsymbol{U}(\boldsymbol{\%})$} & \multicolumn{2}{c|}{$\Delta \boldsymbol{E}(\boldsymbol{\%})$} \\
\cline { 2 - 5 } & $\mathbf{9 5 \%} \mathbf{C I}$ & Avg. & $\mathbf{9 5 \%}$ CI & Avg. \\
\hline 69.4 & -0.507 & -0.295 & -9.87 & -9.36 \\
\hline 65.0 & -0.268 & -0.120 & -5.58 & -5.45 \\
\hline 60.0 & 0.154 & 0.133 & -18.9 & -14.9 \\
\hline
\end{tabular}

\subsection{Performance Comparison between Default and GLA link weights for MPD-aware ETE Schemes}

In this section, we present a direct comparison study, in terms of load-balancing and energy-saving, between the Default and the GLA link weights for the MPD-aware ETE schemes. Table 14 shows the change in load-balancing and energysaving performance when the GLA link weights are used, instead of the Default ones, for the LF-D and MP-D ETE schemes. For the LF-D scheme, GLA is able to improve both the load-balancing and the energy-saving performance over the Default link weights. For the MP-D scheme, GLA is only able to improve the load-balancing performance at the expense of the energy-saving gains. When GLA converges, a final population of solutions, which contains a number of Pareto-optimal solutions, is obtained. Pareto-optimal solutions are solutions, which arise in multi-objective optimization, where one objective cannot be improved at the expense other objectives. In short, Pareto-optimal solutions offer different trade-offs in terms of load-balancing and energy-savings. If the energy-savings performance is prioritized over the loadbalancing performance for the MP-D scheme, it is possible to get values of $-4.11 \%$ and $5.29 \%$ for $\Delta U$ and $\Delta E$ respectively. Therefore, it is possible to get a moderate gain of energy-saving and load-balancing if solutions which do not have close-tooptimal load-balancing performance are chosen. Table 15 shows that it is possible to use GLA-SH to achieve significant improvement in both load-balancing and energy-savings performance over the Default link weights.

\section{Summary}

In this paper, we proposed the Green Load-balancing Algorithm (GLA), which intelligently optimizes IGP link weights in IP backbone networks in order to improve both the load-balancing and energy efficiency of existing ETE schemes. GLA uses a customized multi-objective genetic algorithm to identify the optimized solutions. In addition, two new custom mutation and crossover operators have been designed to improve the performance of the genetic algorithm by enabling the solution space to be searched more efficiently. In order to be able to draw generic conclusions on the energy-efficiency and load-balancing performance of GLA, we selected three diverse existing ETE schemes, LF, MP and TLS, to extensively evaluate the ability of GLA to jointly optimize the load-balancing and energy-efficiency performance of these schemes. Simulations based on the European academic network GÉANT and its real traffic matrices were used to evaluate GLA. GLA was shown to improve the energy efficiency of LF, MP and TLS by $16.1 \%, 1.08 \%$ and $216 \%$ respectively compared to the Default link weight setting scenario. This improvement has been achieved while maintaining near-optimal load-balancing performance as shown through a comparison with IGP-WO. An enhanced version of GLA, GLA-SH, was also designed specifically for TLS. GLASH was able to improve the energy efficiency of TLS by $239 \%$ compared to the Default link weight setting, while maintaining near-optimal load-balancing performance.

Table 14. Performance improvement when GLA link weights are used for LF-D and MP-D ETE scheme compared to Default link weights

\begin{tabular}{|l|l|l|l|l|}
\hline \multirow{2}{*}{$\begin{array}{l}\text { ETE } \\
\text { Scheme }\end{array}$} & \multicolumn{2}{|c|}{$-\Delta \boldsymbol{U}(\%)$} & \multicolumn{2}{c|}{$\Delta \boldsymbol{E}(\%)$} \\
\cline { 2 - 5 } & $\mathbf{9 5 \%}$ CI & Avg. & $\mathbf{9 5 \%}$ CI & Avg. \\
\hline LF-D & 31.4 & 30.5 & 7.61 & 5.42 \\
\hline MP-D & 31.3 & 30.1 & -0.776 & -3.03 \\
\hline
\end{tabular}

Table 15. Performance improvement when GLA-SH link weights are used for TLS-D ETE scheme compared to Default link weights

\begin{tabular}{|l|l|l|l|l|}
\hline \multirow{2}{*}{$\begin{array}{c}\boldsymbol{\alpha}(\boldsymbol{\%}) \\
\end{array}$} & \multicolumn{4}{|c|}{ GLA-SH } \\
\cline { 2 - 5 } & \multicolumn{2}{|c|}{$-\Delta \boldsymbol{U}(\boldsymbol{\%})$} & \multicolumn{2}{c|}{$\Delta \boldsymbol{E}(\boldsymbol{\%})$} \\
\cline { 2 - 5 } & $\mathbf{9 5 \%} \mathbf{C I}$ & Avg. & $\mathbf{9 5 \%} \mathbf{C I}$ & Avg. \\
\hline 69.4 & 23.7 & 23.4 & 59.8 & 55.5 \\
\hline 65.0 & 23.7 & 23.5 & 216 & 202 \\
\hline 60.0 & 23.7 & 23.5 & 244 & 223 \\
\hline
\end{tabular}


We then analysed the Maximum Packet Delay (MPD) performance of the same three ETE schemes. We found that the ETE schemes often exhibit excessive MPD because they are completely oblivious to the increase in packet delay when they put links to sleep to save energy and/or when they optimize the link weights to improve the load-balancing in the network. In light of this observation, we modified the plain ETE schemes to take into account the MPD performance and evaluated their load-balancing and energy-savings performance. We found that it was still possible to save significant energy while achieving near-to-optimal load-balancing in most scenarios and still respect a bound on the maximum packet delay.

The performance of the reduced network topology, obtained by GLA on top of TLS, during single link failures can be guaranteed by using an extended version of TLS called Time-driven Link Sleeping with Single Link Failure Protection (TLSSLFP) published in [9]. GLA can operate with no modification on top of TLS-SLFP and this will be studied in our future work along with our own extension of the LF and MP schemes to support single link failures.

In summary, GLA can be regarded as a very promising approach which is able to further enhance the performance of ETE algorithms while maintaining at the same time the capability of the produced network configurations towards supporting traditional traffic engineering objectives such as load-balancing and MPD.

\section{Acknowledgment}

The research leading to these results has been performed within the UniverSelf project (www.UniverSelf-project.eu) and received funding from the European Community's Seventh Framework Programme (FP7/2007-2013) under grant agreement $\mathrm{n}^{\circ}$ 257513. In addition, this project received support from EU FP7 IRSES EVANS project (PIRSES-GA-2010-269323).

\section{References}

[1] R. Bolla, F. Davoli, R. Bruschi, K. Christensen, F. Cucchietti, and S. Singh, "The potential impact of green technologies in next-generation wireline networks: Is there room for energy saving optimization?" IEEE Commun. Mag., vol. 49, no. 8, pp. 80-86, August 2011.

[2] A. Bianzino, C. Chaudet, D. Rossi, and J. Rougier, “A survey of green networking research,” IEEE Commun. Surveys Tutorials, no. 99, pp. 1-18, 2010 .

[3] R. Bolla, R. Bruschi, F. Davoli, and F. Cucchietti, "Energy Efficiency in the Future Internet: A Survey of Existing Approaches and Trends in EnergyAware Fixed Network Infrastructures," IEEE Commun. Surveys Tutorials, no. 99, pp. 1-22, 2010.

[4] N. Wang, K. Ho, G. Pavlou, and M. Howarth, "An overview of routing optimization for internet traffic engineering," IEEE Commun. Surveys Tutorials, vol. 10, no. 1, pp. 36-56, 2008.

[5] G. Shen and R. Tucker, "Energy-Minimized Design for IP Over WDM Networks," IEEE J. Opt. Commun. Netw., vol. 1, no. 1, pp. 176-186, June 2009.

[6] M. Zhang, C. Yi, B. Liu, and B. Zhang, "GreenTE: Power-Aware Traffic Engineering," in Proc. 2010 of IEEE International Conference on Network Protocols, October 2010.

[7] F. Francois, N. Wang, K. Moessner, and S. Georgoulas, "Optimization for Time-driven Link Sleeping Reconfigurations in ISP Backbone Networks," in Proc. 2012 of IEEE Network Operations and Management Symposium, April 2012.

[8] F. Francois, N. Wang, K. Moessner, and S. Georgoulas, "Optimizing Link Sleeping Reconfigurations in ISP Networks with Off-Peak Time Failure Protection," IEEE Trans. Netw. Service Manag, no. 99, pp. 1-13, 2012.

[9] L. Roberts, “A radical new router," Spectrum, IEEE, vol. 46, no. 7, pp. 34 -39, july 2009.

[10] M. Yamada, T. Yazaki, N. Matsuyama, and T. Hayashi, "Power efficient approach and performance control for routers," in Communications Workshops, 2009. ICC Workshops 2009. IEEE International Conference on, june 2009, pp. 1 -5.

[11] M. Baldi and Y. Ofek, "Time for a "greener" internet," in Communications Workshops, 2009. ICC Workshops 2009. IEEE International Conference on, june 2009, pp. $1-6$.

[12] R. Bolla, R. Bruschi, and A. Ranieri, "Energy-aware equipment for next-generation networks," in Proceedings of the 4th International Conference on Future Internet Technologies, ser. CFI '09. New York, NY, USA: ACM, 2009, pp. 8-11.

[13] M. Mandviwalla and N.-F. Tzeng, "Energy-efficient scheme for multiprocessor-based router linecards," in Proceedings of the International Symposium on Applications on Internet. Washington, DC, USA: IEEE Computer Society, 2006, pp. 156-163.

[14] J. Restrepo, C. Gruber, and C. Machuca, "Energy Profile Aware Routing," in Communications Workshops, 2009. ICC Workshops 2009. IEEE International Conference on, 2009, pp. 1 -5.

[15] A. Cianfrani, M. Listanti, V. Eramo, M. Marazza, and E. Vittorini, "An energy saving routing algorithm for a green ospf protocol," in Proc. IEEE Infocom'10, 2010.

[16] L. Irish and K. Christensen, “A "Green TCP/IP" to reduce electricity consumed by computers,” in Southeastcon '98. Proceedings. IEEE, apr 1998, pp. $302-305$.

[17] R. Bolla, R. Bruschi, A. Cianfrani, and M. Listanti, “Enabling backbone networks to sleep,” IEEE Network, vol. 25, no. 2, pp. 26-31, March-April 2011.

[18] S. Nedevschi, L. Popa, G. Iannaccone, S. Ratnasamy, and D. Wetherall, "Reducing network energy consumption via sleeping and rate-adaptation," in Proceedings of the 5th USENIX Symposium on Networked Systems Design and Implementation, ser. NSDI'08. Berkeley, CA, USA: USENIX Association, 2008, pp. 323-336.

[19] G. Da Costa, J.-P. Gelas, Y. Georgiou, L. Lefevre, A.-C. Orgerie, J.-M. Pierson, O. Richard, and K. Sharma, “The green-net framework: Energy efficiency in large scale distributed systems," in Parallel Distributed Processing, 2009. IPDPS 2009. IEEE International Symposium on, may 2009, pp. $1-8$.

[20] M. Gupta and S. Singh, "Greening of the internet," in Proc. 2003 of SIGCOMM, 2003, pp. 19-26.

[21] L. Chiaraviglio, M. Mellia, and F. Neri, "Reducing power consumption in backbone networks," in Proc. 2009 of the IEEE International Conference on Communications, June 2009, pp. 2298-2303. 
[22] L. Chiaraviglio, M. Mellia, and F. Neri, "Energy-aware backbone networks: A case study," in Proc. 2009 of the IEEE International Conference on Communications, June 2009, pp. 1-5.

[23] A. Cianfrani, V. Eramo, M. Listanti, M. Polverini, and A. Vasilakos, "An ospf-integrated routing strategy for qos-aware energy saving in ip backbone networks," IEEE Trans. Netw. Service Manag., no. 99, pp. 1-14, 2012.

[24] W. Fisher, M. Suchara, and J. Rexford, "Greening backbone networks: reducing energy consumption by shutting off cables in bundled links," in Proc. 2010 of ACM SIGCOMM workshop on Green networking, 2010, pp. 29-34.

[25] M. Shen, H. Liu, K. Xu, N. Wang, and Y. Zhong, "Routing on demand: toward the energy-aware traffic engineering with OSPF," in Proc. 2012 of the 11th International IFIP TC6 Conference on Networking, 2012, pp. 232-246.

[26] N. Vasic and D. Kostic, "Energy-aware traffic engineering," in Proc. 2010 of International Conference on Energy-Efficient Computing and Networking, 2010, pp. 169-178.

[27] N. Vasic, D. Novakovic, S. Shehar, P. Bhurat, M. Canini, and D. Kostic, "Identifying and Using Energy-Critical Paths," in Proc. 2011 of ACM CoNEXT, December 2011.

[28] C. Panarello, A. Lombardo, G. Schembra, L. Chiaraviglio, and M. Mellia, "Energy saving and network performance: a trade-off approach," in Proc. 2010 of International Conference on Energy-Efficient Computing and Networking, 2010, pp. 41-50.

[29] A. Bianzino, L. Chiaraviglio, and M. Mellia, "Grida: A green distributed algorithm for backbone networks," in Proc. 2011 of IEEE GreenCom, September 2011, pp. 113-119.

[30] M. Charalambides, D. Tuncer, L. Mamatas, and G. Pavlou, "Energy-Aware Adaptive Network Resource Management," in Proc. 2013 of the IFIP/IEEE Integrated Management Symposium, May 2013.

[31] A. Lakhina, K. Papagiannaki, M. Crovella, C. Diot, E. D. Kolaczyk, and N. Taft, "Structural analysis of network traffic flows," in Proc. 2004 of the Joint International Conference on Measurement and Modeling of Computer Systems, 2004, pp. 61-72.

[32] A. Nucci, A. Sridharan, and N. Taft, "The problem of synthetically generating IP traffic matrices: initial recommendations," SIGCOMM Comput. Commun. Rev., vol. 35, no. 3, pp. 19-32, July 2005.

[33] M. Roughan, A. Greenberg, C. Kalmanek, M. Rumsewicz, J. Yates, and Y. Zhang, "Experience in measuring backbone traffic variability: models, metrics, measurements and meaning," in Proc. 2002 of ACM SIGCOMM Workshop on Internet measurment, 2002 , pp. 91-92.

[34] S. Uhlig, B. Quoitin, J. Lepropre, and S. Balon, "Providing public intradomain traffic matrices to the research community," SIGCOMM Comput. Commun. Rev., vol. 36, pp. 83-86, January 2006.

[35] B. Fortz and M. Thorup, "Increasing Internet Capacity Using Local Search," Comput. Optim. Appl., vol. 29, pp. 13-48, October 2004.

[36] K. Deb, A. Pratap, S. Agarwal, and T. Meyarivan, “A fast and elitist multiobjective genetic algorithm: NSGA-II,” IEEE Trans. Evol. Comput., vol. 6, no. 2, pp. 182-197, April 2002.

[37] "The totem traffic engineering toolbox," http://totem.run.montefiore.ulg.ac.be, online. Assessed: Jan. 2014.

[38] "Cisco 12000 series packet over sonet/sdh (pos) line cards," http://www.cisco.com/en/US/prod/collateral/routers/ps6342/product_data_sheet0900aecd803fd7b9.pdf, online. Assessed: Jan. 2014.

[39] B.-Y. Choi, S. Moon, Z.-L. Zhang, K. Papagiannaki, and C. Diot, "Analysis of point-to-point packet delay in an operational network," Comput. Netw., vol. 51, no. 13, pp. 3812-3827, September 2007. 